\title{
REGENERATED CELLULOSE/GRAPHENE COMPOSITE FIBERS WITH ELECTROCONDUCTIVE PROPERTIES
}

\author{
Kulpinski Piotrr,*, Jeszka Jeremiasz K. ${ }^{1}$, Malolepszy Artur², Stobinski Leszek ${ }^{2,3}$ \\ 1 Department of Mechanical Engineering, Informatics and Chemistry of Polymer Materials, Lodz University of Technology, \\ Zeromskiego 116, 90-924 Lodz, Poland \\ 2 Faculty of Chemical and Process Engineering, Warsaw University of Technology, Warynskiego 1, 00-654 Warszawa, Poland \\ 3 NANOMATERIALS LS (www.nanomaterials.pl), Wyszogrodzka 14/38, 03-337 Warsaw, Poland \\ ${ }^{*}$ Corresponding author. E-mail: piotr.kulpinski@p.lodz.pl
}

\begin{abstract}
:
Conductive cellulose fibers may find application in producing antistatic materials and fibrous electronic elements for smart textiles (textronics). In this paper, we present a method of fabrication of cellulose fibers modified with the reduced graphene oxide ( $\mathrm{GGO}$ ) and graphene oxide (GO). The fibers were obtained by using $\mathrm{N}$-methylmorpholine$\mathrm{N}$-oxide (NMMO) as a direct solvent, adding dispersion of GO during the cellulose dissolution process. In the next step, the GO enclosed in the fibers was reduced for ca. half an hour at $90^{\circ} \mathrm{C}$ with the excess of water solution of hydrazine to obtain fibers containing $\mathrm{rGO}$. The viscosity of the spinning solution increased when GO was added; however, the difference is important at low shearing rates but decreases at high shearing rates, similar to that used in the process of fiber spinning. Cellulose fibers containing 3, 4, 6, and 10\% w/w of rGO were obtained. Fiber morphology was studied using electron microscopy. The results of the electrical properties' measurements showed that the conductivity of modified fibers strongly depends on the concentration of rGO. At $10 \%$ rGO conductivity was $9 \times 10^{-3} \mathrm{~S} / \mathrm{cm}$. The mechanical properties of the obtained fibers were slightly changed by the presence of GO and rGO. Tenacity and elongation at break decreased with the increase in the content of GO and rGO in the fibers but remain at an acceptable level from the textiles processing point of view.
\end{abstract}

\section{Keywords:}

Cellulose fibers, graphene oxide, reduced graphene oxide, electroconductive fibers, NMMO

\section{Introduction}

Cellulose is a natural fiber-forming polymer. Although the chemical fiber market was dominated by synthetic polymers for the last 40 years, recently a growing interest in renewable and biodegradable materials once again shed light on cellulosederived fibers [1]. Cellulose fibers find numerous applications due to their good mechanical properties and low price. Their conductivity under ambient conditions is high enough to avoid significant contact charging but far too low to be used as conductive fibers in textile-based electronic elements, such as antennae, sensors, signal lines, and solar batteries. This field of technology is known as textronics [2] or smart textiles [3].

Many methods have been proposed to increase the conductivity of fibers or textiles [3, 4]. The first possibility is to use an admixture of conductive fibers (e.g., metallic or metallized polymer fibers or bulk conductive polymer fibers) to the basic textile material [3]. The most advantageous situation is when conductive fibers have very similar mechanical and processing properties as the main fibers of the textile. The second possibility is to make the fiber surface conductive. A variety of materials has been proposed for this purpose [4]. The third method is the modification of the bulk conductivity of the polymer fibers by adding a suitable conductive filler to the polymer before or during the spinning dope preparation process.
Graphite/graphene flakes have been very popular conductive additives for a long time because of their unique mechanical, electrical, and chemical properties [5]. However, it is difficult to obtain a good dispersion of graphene flakes in a polymer matrix, particularly in hydrophilic ones like cellulose because of its hydrophobicity. Graphite oxide (recently referred to as graphene oxide) (GO), obtained by oxidation of graphite is hydrophilic and can be used as a graphene precursor because it can be reduced again to form (defected) graphene sheets [6-8]. GO behaves like a large amphiphilic macromolecule with hydrophilic edges and a more or less hydrophobic basal plane $[7,8]$. It is possible to achieve a truly molecular-level $\mathrm{GO}$ dispersion when the aqueous system is used for the GO dispersion. The functional groups formed during graphite oxidation allow GO to be easily dispersed in the hydrophilic polymers to form uniform composites due to creation of hydrogen bonds between $\mathrm{GO}$ and the polymer [7-9].

It is also possible to reduce GO inside the composite to obtain graphene flakes, that is, reduced graphene oxide ( $\mathrm{rGO}$ ) even in situ in the composite $[7,8]$. To reduce $G O$ to rGO, chemical reducing agents, such as hydrazine, ascorbic acid, and many others can be used as well as UV radiation or high temperature [6-8]. Although the graphene thus obtained is of poor quality, with many defects, it is relatively well conductive bringing the electrical conductivity to the obtained composites. Several polymers, including poly(vinyl alcohol) [10], chitosan [11], 
cellulose [12-16], poly(vinylidene fluoride) [17], and polylactide [18] have been successfully used to prepare GO/polymer composites.

The Lyocell process of manufacturing cellulose fibers [19] is an interesting alternative for conventional methods, such as the viscose process or cuprammonium process, since it is free from their most serious drawbacks. Besides, this technology is characterized by a short and simple production cycle and it is environmentally friendly, because of using non-toxic solvent $\mathrm{N}$-methylmorpholine-N-oxide hydrate (NMMO) with its closed circulation. This technology also allows the production of composite fibers by simple incorporation of various additives including conductive materials into the spinning solution [2022].

Preparing the first conductive composite cellulose fibers by the Lyocell method was reported by Meister et al. [23]. They used a carbon black (CB) as a filler and showed that cellulose/CB/ NMMO solutions can be spun even at high CB content. They obtained conductive fibers but did not study the fiber properties in detail.

The present work aimed to obtain cellulose fibers by a drywet spinning method with the use of NMMO as a solvent of cellulose. The fibers were modified with graphene oxide, which was then reduced using an aqueous hydrazine solution. The influence of GO concentration and time of GO reduction on the electrical and mechanical properties of the obtained fibers were investigated. This kind of innovative material seems to be interesting and due to its unique properties find many potential applications, for example, in textronics and protection cloths (with antistatic effect).

\section{Experimental}

\subsection{Materials}

Cellulose pulp (Rayonier®) with a polymerization degree of 1236 was used. A $50 \%$ aqueous solution of NMMO manufactured by the Huntsman Corporation was used as a direct solvent. Tenox PG (propyl ester of gallic acid) (Sigma®) was used as an antioxidant. The water dispersion of GO used as a modifier was prepared by Nanomaterials LS Company and Graphene Laboratory at Warsaw Technical University, with the method described in [24]. The water solution of hydrazine (Aldrich) was used for the reduction of GO.

\subsection{Methods}

The morphology of the bulk of the fibers was investigated by transmission electron microscopy (TEM) and high-resolution electron microscopy (HREM) using Tecnai Super Twin FEG $(200 \mathrm{kV})(\mathrm{FEI}, \mathrm{USA})$ in the accredited analytical laboratory of electron microscopy (L-2) of the Polish Academy of Sciences in Krakow. Scanning electron microscope (SEM) images of the fiber surface were made using the Vega3-SBU TESCAN microscope (Czech Republic). Elemental analysis was performed using the Vario Micro Cube apparatus (Elemental Analisensysteme $\mathrm{GmbH}$ ).

Rheological measurements were performed using the MCR301 apparatus made by Anton Paar (Germany). The measurements were carried out in rotary mode in the cone-plate configuration. The cone diameter was $25 \mathrm{~mm}$ and the cone angle was $2^{\circ}$.

Electrical conductivity was measured on bundles of ca. 50 fibers provided with electrodes made using silver paint. The distance between the electrodes was ca. $3 \mathrm{~cm}$. The exact number of fibers and the average cross-section determined under the optical microscope were used to calculate the total bundle width. Source-Meter Unit Keithley 2614B was used to provide the desired voltage and to measure electric current. Currentvoltage characteristics have shown that the conductivity of the obtained fibers is ohmic, at least up to voltages $3 \times 10^{3} \mathrm{~V} / \mathrm{m}$, that is, in the range in which the measurements were made.

The mechanical properties of fibers were checked on a Zwick Z2.5/TN1S tensile testing machine following ISO standard PN-EN ISO 5079:1995. The linear density of the fibers was measured according to ISO standard ISO 1973:1995.

\subsubsection{Preparation of cellulose fibers modified with GO}

The method of obtaining the cellulose fibers was similar as described elsewhere [25]. The cellulose solutions in NMMO containing $8 \%$ of cellulose were obtained in high-efficiency laboratory-scale IKAVISC kneader type MKD 0.6-H60. The mixture of $50 \%$ of water solution of $\mathrm{NMMO}, 1 \%$ water dispersion of $\mathrm{GO}$, and cellulose was heated to $115^{\circ} \mathrm{C}$ under low pressure $(120 \mathrm{hPa})$, and an appropriate amount of water was removed from the system. The process was continued until a homogeneous solution of cellulose was obtained. The water dispersion of GO was added at the beginning of the described process in such amount to obtain $6,8,12$, and $20 \%$ of modifier in the cellulose fibers.

The cellulose fibers were made using a dry-wet spinning method on a laboratory-scale piston - spinning device with spinneret with 18 orifices of $0.4 \mathrm{~mm}$ diameter. The fibers were then washed and divided into two parts. One was dried at ambient temperature and the other, after the removal of the excess of water, was immersed in an aqueous hydrazine solution to reduce $\mathrm{GO}$ to rGO. Cellulose fibers without the addition of GO were also spun at the same conditions and used as a reference sample.

\subsubsection{Reduction of graphene oxide}

To obtain the cellulose fibers modified with $\mathrm{rGO}$ the reduction of $\mathrm{GO}$ in the fibers was carried out. Cellulose/GO fibers not yet dried after the spinning process were immersed in the $5 \%$ aqueous hydrazine solution at $90^{\circ} \mathrm{C}$. To estimate the optimal time of the reduction reaction the fibers were kept in the reduction system for, 15, 30, 60, 180 and $360 \mathrm{~min}$. respectively. The solution of hydrazine was used in the about 10 times molar excess of hydrazine to the oxygen introduced into GO. After the reduction reaction, the fibers were washed with water for $24 \mathrm{~h}$ 
and then dried at ambient temperature. During this process, initially brown fibers turned black. The rGO content in the fibers was calculated assuming that during the reduction process about $50 \%$ of modifier weight was lost.

\section{Results and discussion}

\subsection{Elemental analysis}

To estimate the progress of the reduction reaction in the cellulose fibers containing $12 \% \mathrm{w} / \mathrm{w}$ of $\mathrm{GO}$, the elemental analysis was done. The results are shown in Table 1.

According to the presented results, the reaction is completed after $30 \mathrm{~min}$. It can be seen that the steady-state (within experimental error $0.07 \%$ ) is reached after $30 \mathrm{~min}$, at most $60 \mathrm{~min}$. Further extension of the reaction time does not significantly increase the carbon content in the tested fibers. This observation is also confirmed by the results of measuring the electrical conductivity of the fibers. The preliminary studies show that other reducing agents as ascorbic acid can also be used, but the hydrazine was the most effective one.

\subsection{Rheological properties of spinning dopes}

The viscosity of spinning solutions influences spinning conditions and the properties of the fibers obtained. Solutions of cellulose in NMMO are known to exhibit non-Newtonian properties, i.e., viscosity depends on shear rate, not only on the polymer concentration and temperature. Figure 1 shows flow curves for two solutions containing different quantities of $\mathrm{GO}$ at two temperatures close to the spinning temperature.

All investigated solutions show a characteristic typical for polymer materials: at low shear rates the viscosity is shear independent (first Newtonian range); for higher shear rates the viscosity decreases approximately following the power law (shear thinning range). In the first Newtonian range, the addition of GO brings about an increase of viscosity up to 6 times for $20 \%$ GO. However, in the shear-thinning range the viscosity of solutions containing GO decreases faster, so at ca. $30 \mathrm{~s}^{-1}$ the viscosity of all systems is practically the same. In all solutions, viscosity increases with the increase in temperature (as expected) up to shearing rate $30 \mathrm{~s}^{-1}$, but the difference between viscosities at $90^{\circ} \mathrm{C}$ and $110^{\circ} \mathrm{C}$ decreases because of a stronger shear thinning.

Table 1. The results of the elemental analysis of cellulose fibers containing $12 \%$ of GO after different times of reduction reaction

\begin{tabular}{|c|c|c|}
\hline $\begin{array}{c}\text { Reaction time } \\
{[\mathrm{min}]}\end{array}$ & $\begin{array}{c}\text { Carbon content } \\
{[\% \text { w/w] }}\end{array}$ & $\begin{array}{c}\text { Hydrogen } \\
\text { content [\% w/w] }\end{array}$ \\
\hline 0 & 40.17 & 5.98 \\
\hline 15 & 42.17 & 6.17 \\
\hline 30 & 43.98 & 6.78 \\
\hline 60 & 43.91 & 6.91 \\
\hline 360 & 43.90 & 6.98 \\
\hline
\end{tabular}

\subsection{Fiber morphology}

The scanning electron microscopy image of the fibers with $20 \%$ w/w of GO is shown in Figure 2. The surface of fibers shows a typical morphology of lyocell fibers, which is not significantly modified by the addition of GO or by GO reduction process $(6 \mathrm{~h}$ in hydrazine solution). GO and rGO sheets cannot be seen at this magnification.

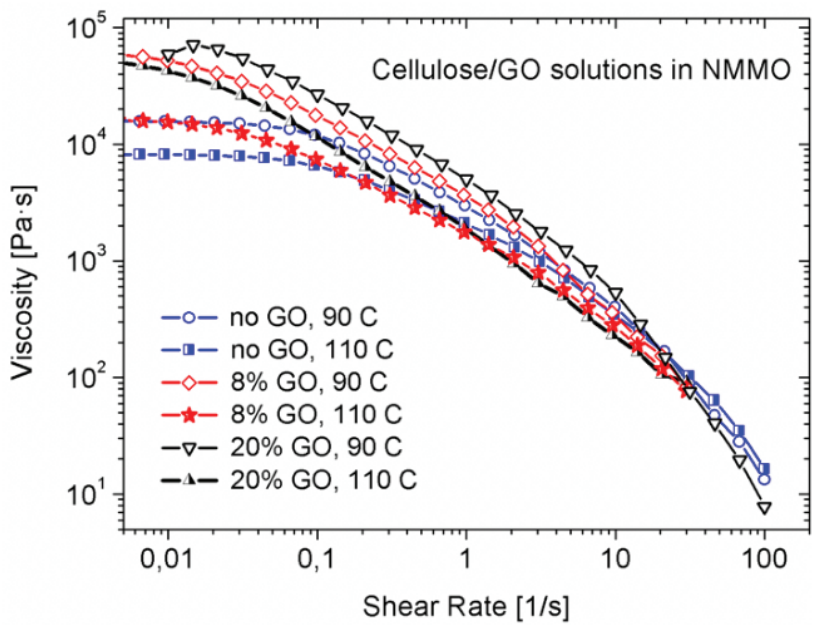

Figure 1. Shear rate dependence of viscosity for cellulose solution in NMMO without $\mathrm{GO}$ and containing different amounts of $\mathrm{GO}$ at $90^{\circ} \mathrm{C}$ and $110^{\circ} \mathrm{C}$. GO content is calculated for cellulose.
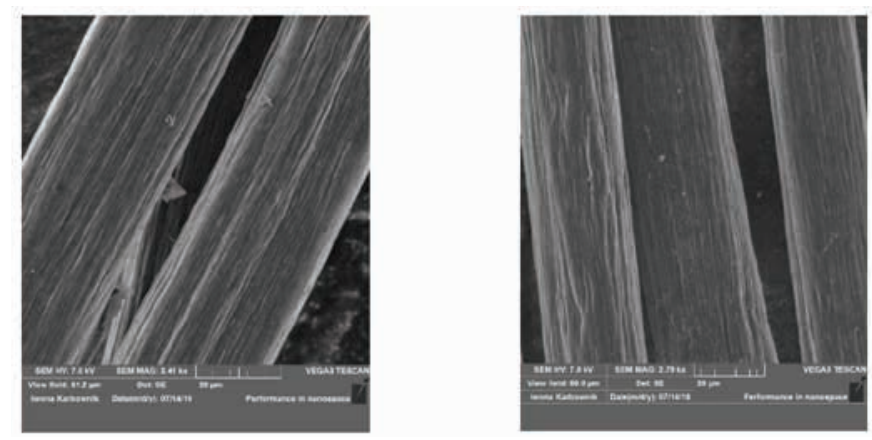

Figure 2. (a) Scanning electron microscopy image of the fibers with $20 \%$ w/w of GO and (b) rGO.
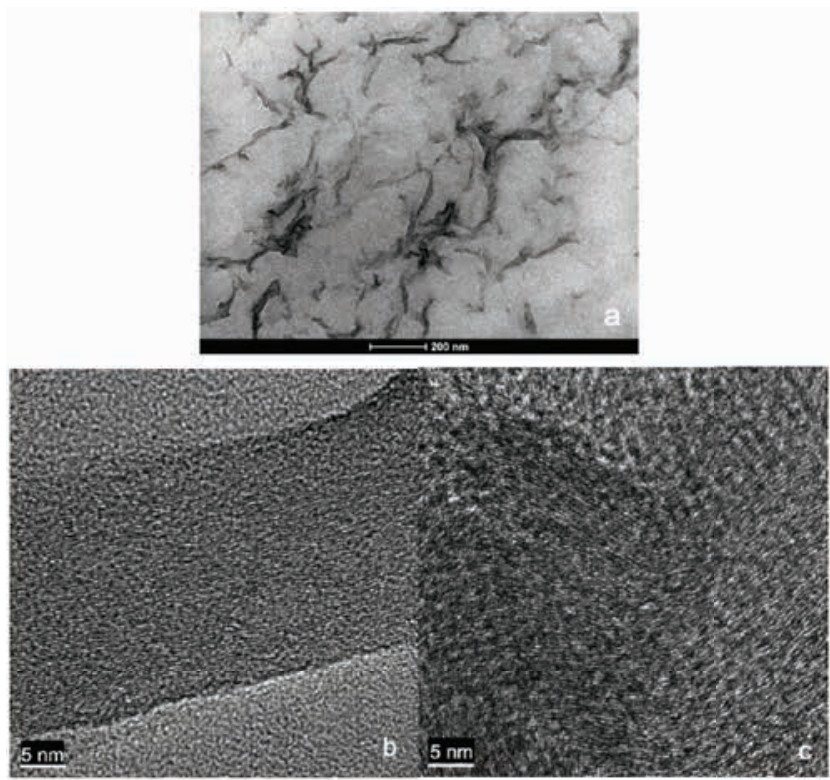

Figure 3. (a) TEM electron microscopy images of the cross-sections of fibers with 20 wt.\% of rGO, (b) HREM for fibers, and (c) HREM rGO. 
Transmission electron microscopy of thin slices of the fibers (Figure 3) reveals the presence of graphene flakes in the bulk. One can estimate their thickness to be 10-20 nm. HREM images show the layered structure of the plane flakes; however, the estimation of the graphene layer stacking, which is expected to be $0.89 \mathrm{~nm}$ in $\mathrm{GO}$ and $0.37 \mathrm{~nm}$ in rGO, is not reliable because of their disordered structure.

\subsection{Electrical properties}

Fibers with $\mathrm{GO}$ are insulators for all the investigated contents of $\mathrm{GO}$. After the reduction of $\mathrm{GO}$ using hydrazine the fibers become conductive. Figure 4 shows the dependence of conductivity on the rGO content. One can see that the experimental points fit well theoretical predictions of the percolation model $\sigma=\sigma$ 。 $\left(p-p_{c}\right)^{t}$ where $\sigma-$ material conductivity, $\sigma_{o}$ - conductivity of the conductive phase, $p$-concentration of the conductive phase, $p_{c}$ - percolation threshold, and $t-$ critical exponent $[26,27]$.

The parameters determined from the fitting of this equation to the experimental results are shown in Figure 4. Percolation threshold $p_{c}=2.46 \mathrm{wt}$. $\%$ is higher than determined for cellulose films with GO and some similar composites, however in welloriented fibers $p_{c}$ is usually higher as compared with non-oriented

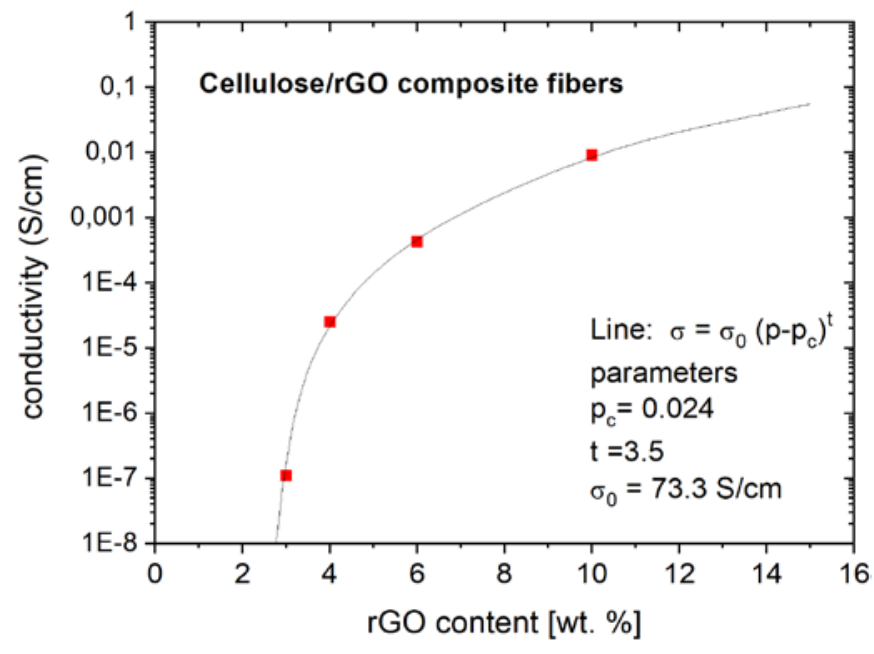

Figure 4. Dependence of electrical conductivity on the rGO content in cellulose fibers. The line represents theoretical values obtained using the percolation equation with the parameters shown in the inset. composites because many contacts between conductive additive sheets are broken and the distances between them measured in the direction of the fiber axis increase during the drawing process. Parameter $\sigma_{o}$ represents conductivity of the conductive phase (in our case $\mathrm{rGO}$ ) and it has a reasonable value of $73.3 \mathrm{~S} \times \mathrm{cm}^{-1}$ similar to $24 \mathrm{~S} \times \mathrm{cm}^{-1}\left(2,400 \mathrm{~S} \times \mathrm{m}^{-1}\right)$ reported for compressed pellets of rGO [28]. Critical exponent $t$ is higher than theoretically predicted and found from simulations for bond- or site-percolation on 3D networks (1.65). Continuum models and mean-field theory yield higher values (2.5-3) [26, 27]. These models seem however less appropriate to the case of the network of highly conductive filaments. However, taking into account the possible non-homogeneous dispersion and orientation of the defected rGO flakes the value $t=3.5$ obtained in this work seems reasonable.

\subsection{Mechanical properties}

The mechanical properties represent the most important features of the fibers. The tenacity of fibers and the elongation at the break as well as some other properties strongly depend not only on the degree of polymerization of the cellulose or the conditions of the spinning process but also depend on the kind, concentration, and degree of dispersion of the introduced modifiers. The acceptable level of the mechanical properties of the fibers is also important from the textile manufacturing point of view. When a textile product is manufactured, the fibers should withstand several processes as, for example, yarn formation, knitting or waving. During these processes, the fibers undergo bending, stretching and twisting, which to some extend deteriorate their mechanical properties. Thus, good mechanical properties of the fibers are an important factor for their processing. Application of fibers with low mechanical parameters means that during fiber processing the fibers break, which causes many technical problems and errors in the final product. In extreme cases, fibers may be completely unsuitable for textile processing. From a practical point of view, it is thus very important to obtain fibers with the best possible mechanical parameters.

The influence of GO, as well as rGO on the mechanical properties, and the linear density of the fibers were studied. The results are collected in Table 2.

Table.2. The linear density and mechanical properties of fibers with different content of the modifiers

\begin{tabular}{|c|c|c|c|c|c|}
\hline Modifier & Concentration (\%) & Linear density (tex) & Tenacity (cN/tex) & Modulus (cN/tex) & Elongation at break (\%) \\
\hline \multirow{3}{*}{ No } & 0 & 1.16 & 30.65 & 1,581 & 11.6 \\
\hline \multirow{3}{*}{ GO } & 6 & 1.69 & 25.24 & 1,470 & 11.9 \\
\cline { 2 - 6 } & 8 & 1.35 & 22.03 & 1,252 & 11.0 \\
\cline { 2 - 6 } & 12 & 1.43 & 19.57 & 1,167 & 11.8 \\
\hline \multirow{3}{*}{ rGO } & 20 & 1.67 & 13.76 & 975 & 1.9 \\
\cline { 2 - 7 } & 3 & 1.32 & 26.80 & 1,597 & 10.9 \\
\cline { 2 - 6 } & 4 & 1.31 & 22.54 & 1,233 & 11.5 \\
\hline
\end{tabular}


It can be seen that the addition of graphene leads to an increase in the linear density of the fibers; the higher the linear density is, the higher will be the graphene content. Since all the fibers' spinning conditions were the same, the result seems to be related to the changing of spinning dope viscosity. The higher the GO content in the spinning solution was, the greater forces were applied to extrude it through the spinneret. The reduction of the GO brings about some decrease in the linear density which is related to a decrease of the oxygen content. In fact, about half of the weight of the modifier is removed during the reduction process. Initial modulus of the fibers with GO or rGO systematically decreases with increasing additives content and is slightly lower for rGO except for fibers with the lowest rGO content $(3 \%)$ for which the value is even somewhat higher than for plain cellulose fibers (however within experimental error). It is, however, the opposite tendency as that observed by Mahmoudian et al. [16] on adding graphene nanoplatelets (GNP) to the lyocell fibers. The modulus of their fibers increases with graphene loading. It should, however, be noted that these authors used microcrystalline cellulose of low degree of polymerization not suitable to obtain good quality fibers and thus the properties of their plain cellulose fibers are poor. Their pure cellulose fibers have modulus 463 , that is, more than three times lower than ours and at $2 \%$ of graphene reaches $750 \mathrm{cN} / \mathrm{tex}$, still lower than we have at $10 \% \mathrm{w} / \mathrm{w}$ of rGO. In other words, poor mechanical properties of fibers are improved by a small number of graphene nanoplatelets, but it is not clear what would happen at higher loadings.

Tenacity and the elongation at break of cellulose fibers decrease when graphene is present both in its reduced or oxidized form (Figures 5 and 6, respectively). The decrease is higher for the higher additive content. This finding is in agreement with a general observation that the addition of non-fiber forming additive results in a deterioration of the mechanical properties of the fibers $[20,21,29]$. The presence of particles or molecules of the modifier decreases the orientation and interactions between the cellulose macromolecules which makes the fibers strong. Moreover, it is the crystalline phase that is mainly responsible for the tenacity of the fibers. The modifier (reduced or not) is located in the amorphous phase, thus it has a limited effect on the mechanical parameters of the fibers as long as its concentration is not too high.

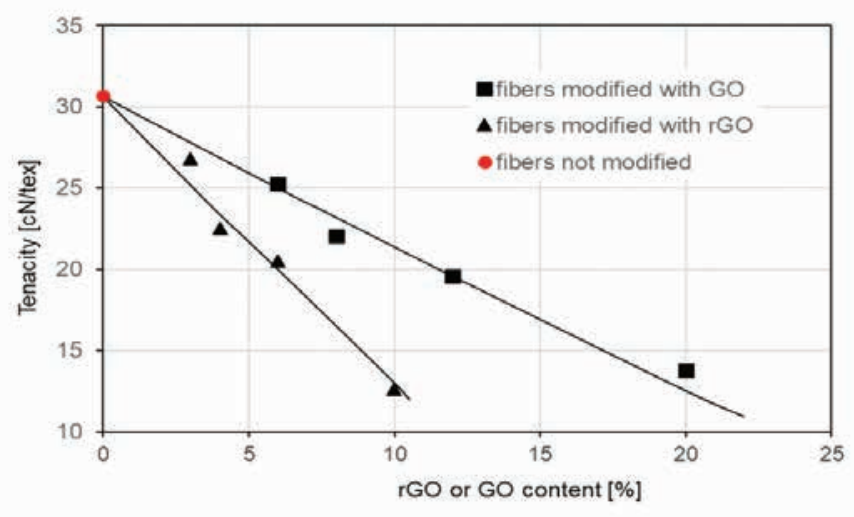

Figure 5. Dependence of tenacity on the GO or rGO content in cellulose fibers.
It is, however, again the opposite tendency as that observed by Mahmoudian et al. [16]. They report tenacity of $23.1 \mathrm{cN} / \mathrm{tex}$ for non-modified cellulose fiber, while we have $30.6 \mathrm{cN} / \mathrm{tex}$ and $38.4 \mathrm{cN} /$ tex at $2 \%$ of GNP, and $26.8 \mathrm{cN} /$ tex at $3 \%$ of $\mathrm{rGO}$. It is again not clear what would happen at higher loadings. It should be emphasized that in the case of fibers containing $20 \%$ of GO, the tenacity decreases to about $55 \%$ of the tenacity of plain cellulose fibers. Most likely, very good dispersion of GO in the polymer matrix and the presence of several hydrophilic groups keeps the fiber tenacity at a relatively high level. Surprisingly, the reduction of the modifier, that is, elimination of the groups that can form hydrogen bonds with cellulose macromolecules does not cause a decrease in strength. For GO contents of 6 , 8 , and $12 \%$, the reduction causes a slight increase in tenacity. Only in the case of the fibers with the highest content, that is, $20 \%$, the GO reduction causes a decrease in tenacity by about $8.7 \%$.

Elongation at break of composite cellulose fibers (Figure 6) practically does not change when small amounts of GO or rGO are added (10.5 and $10.9 \%$ for pure cellulose fibers and $6 \%$ of rGO, respectively) but falls at high additive content to 7.9 or $6.5 \%$ for $\mathrm{GO}$ and $\mathrm{rGO}$, respectively. This is also a typical consequence of adding solid additives to polymers (also to cellulose fibers [25]). Generally, it is the amorphous phase which is mainly responsible for the value of elongation at break. Because of the presence of GO in the fiber (in the amorphous phase), results in the formation of hydrogen bonds between $-\mathrm{OH}$ groups in cellulose macromolecules and $-\mathrm{OH},-\mathrm{COOH}$ or other groups in $\mathrm{GO}$, the value of elongation at break of the composite fibers decreases initially relatively slowly with the increase of GO content.

On the other hand in many cases, especially at high modifier concentration, a decrease of elongation at break is due to non-homogeneities of the modifier dispersion, which act as macroscopic defects. In [16], elongation is reported to decrease when graphene is added, being 11.3 and $8.8 \%$ for non-modified fibers and fibers with $2 \%$ of GNP, respectively, so the tendency is similar.

The difference between the mechanical properties of fibers before and after the reduction of GO is generally small (Figures 5 and 6$)$. It is somewhat surprising, taking into account that

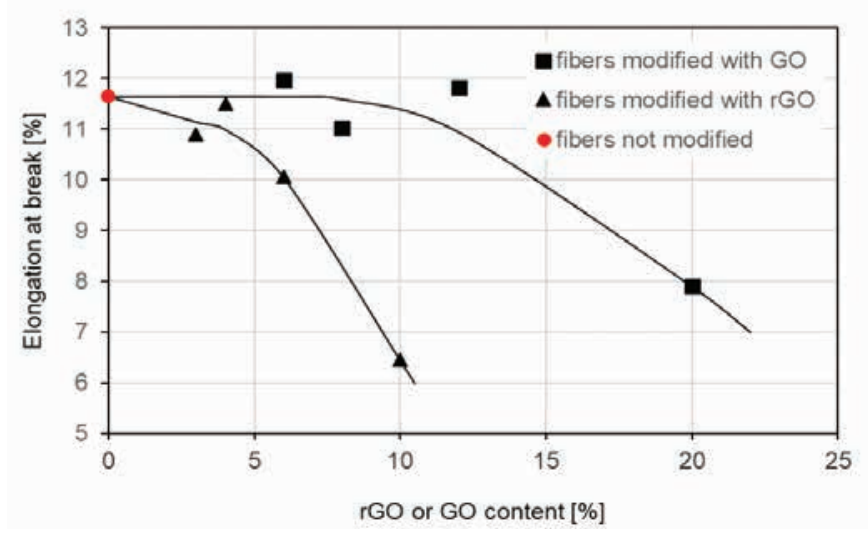

Figure 6. Dependence of elongation at the break on the GO or rGO content in cellulose fibers. 
the presence of groups that can form hydrogen bonds with cellulose leads to good adhesion between cellulose and GO. In rGO (and GNP), such groups are practically absent, so there can be little or no hydrogen bonds and the adhesion between cellulose and graphene sheets is weaker. However, in contrast, during the $\mathrm{GO}$ reduction the double bonds are re-formed and the number of defects is reduced making the graphene sheet stronger. This effect can compensate for weaker adhesion to some extent.

\section{CONCLUSIONS}

Cellulose fibers containing $\mathrm{GO}$ or graphene ( $\mathrm{rGO}$ ) were obtained using NMMO as a direct solvent. Hydrophilic properties of GO make possible homogeneous dispersion of the additive in the spinning dope and later in the fibers being formed. Mechanical properties of such fibers are similar to cellulose fibers formed under the same conditions without GO. Only at high loading $(20 \% \mathrm{w} / \mathrm{w})$ tenacity and modulus are lower. Fibers modified with $\mathrm{GO}$ are non-conducting. $\mathrm{GO}$ in such fibers can be reduced in situ to obtain conductive rGO rendering the fibers in which the concentration exceeds the percolation threshold $2.4 \%$ $\mathrm{w} / \mathrm{w}$ electrically conductive. To obtain fibers modified with $\mathrm{rGO}$, the reduction of $\mathrm{GO}$ in aqueous hydrazine solution was performed, but preliminary studies show that other reducing agents can also be used. Based on elemental analysis and electrical conductivity measurements, it was found that the GO reduction reaction was completed after c.a. $30 \mathrm{~min}$. Maximum conductivity $9 \times 10^{-3} \mathrm{~S} \times \mathrm{cm}^{-1}$ was obtained at $10 \% \mathrm{w} / \mathrm{w}$ of $\mathrm{rGO}$. Mechanical properties of fibers with rGO are similar or slightly worse than those of cellulose fibers with $\mathrm{GO}$ before reduction (the additive content by weight is lower after reduction). In summary, conductive lyocell cellulose fibers can be obtained in an easy two-step procedure using GO containing fibers as an intermediate step to achieve homogeneous dispersion of hydrophobic graphene in a hydrophilic cellulose matrix.

\section{ACKNOWLEDGMENTS}

Financial support from the National Science Centre grant $2012 / 07 / B / S T 8 / 03698$ is gratefully acknowledged.

\section{References}

[1] Woodings, C. (2001). Application development. In: Wooding, C. (Ed.). Regenerated cellulose fibers (1st ed.). Woodhead Publishing Ltd (Cambridge).

[2] Gniotek, K., Krucinska, I. (2004). The basic problems of texstronics. Fibers and Textiles in Eastern Europe, 12(2), 13-16.

[3] Matteo Stoppa, M., Alessandro Chiolerio, A. (2014). Wearable Electronics and Smart Textiles: A Critical Review. Sensors, 14, 11957-11992.

[4] Holme, I., McIntyre, J. E., Shen, Z. J. (1998). Electrostatic charging of textiles. Textile Progress, 28, 1-85.
[5] Sengupta, R., Bandyopadhyay, M., Bhattacharya, S., Bhowmick, A. K. (2011). A review on the mechanical and electrical properties of graphite and modified graphite reinforced polymer composites. Progress in Polymer Science, 36(5), 638-670.

[6] Pei, S., Hui-Ming Cheng, H.-M. (2012) The reduction of graphene oxide. Carbon, 50, 3210-3228.

[7] Lawal A. T., (2019), Graphene-based nano composites and their applications. A review. Biosensors and Bioelectronics, 141, 111384.

[8] Kesong Hu, K., Kulkarni, D. D., Choi, I., Tsukruk, V. V. (2014). Graphene-polymer nanocomposites for structural and functional applications. Progress in Polymer Science 39(11), 1934-1972.

[9] Alemour, B., Yaacob, M., Lim, H. N., Hassan, M. R. (2018). Review of electrical properties of graphene conductive composites. International Journal of Nanoelectronics and Materials, 11(4), 371-398.

[10] Putz, K. W., Compton, O. C., Palmeri, M. J., Nguyen, S. T., Brinson, L. C. (2010). High-nanofiller-content graphene oxide-polymer nanocomposites via vacuum-assisted selfassembly. Advanced Functional Materials, 20, 3322-3329.

[11] Yang, X., Tu, Y., Li, L., Shang, S., Tao, X. (2010). Welldispersed chitosan/graphene oxide nanocomposites. ACS Applied Materials \& Interfaces, 2, 1707-1713.

[12] Han, D., Yan, L., Chen, W., Li, W., Bangal, P. R. (2011). Cellulose/graphite oxide composite films with improved mechanical properties over a wide range of temperature. Carbohydrate Polymers, 83, 966-972.

[13] Zhang, X., Liu, X., Zheng, W., Zhu, J. (2012). Regenerated cellulose/graphene nanocomposite films prepared in DMAC/LiCl solution. Carbohydrate Polymers, 88, 26-30.

[14] Zhang, X., Liu, X., Zheng, W., Zhu, J., (2012). Regenerated cellulose/graphene nanocomposite films prepared in DMAC/LiCl solution. Carbohydrate Polymers 88, 26-30.

[15] Feng, Y., Zhang, X., Shen, Y., Yoshino, K., Feng, W. (2012). A mechanically strong, flexible and conductive film based on bacterial cellulose/graphene nanocomposite. Carbohydrate Polymers, 87, 644-649.

[16] Mahmoudian, S., Sazegar, M. R., Afshari, N., Wahit, M. $U$. (2017). Graphene reinforced regenerated cellulose nanocomposite fibers prepared by lyocell process. Polymer Composites, 38(S1), 81-88.

[17] Eswaraiah, V., Balasubramaniam, K., Ramaprabhu, S. (2012). One-pot synthesis of conducting graphenepolymer composites and their strain sensing application. Nanoscale, 4, 1258.

[18] Hu, C., Li, Z., Wang, Y., Gao, J., Dai, K., et al. (2017). Comparative assessment of the strain-sensing behaviors of polylactic acid nanocomposites: Reduced graphene oxide or carbon nanotubes. Journal of Materials Chemistry C, 5, 2318.

[19] White, P., (2001). Lyocell: the production process and market development. In: Wooding C. (Ed.). Regenerated cellulose fibers (1st ed.). Woodhead Publishing Ltd (Cambridge).

[20] Kaszuwara, W., Rubacha, M., Leonowicz, M., Kulpinski, P., Laszkiewicz, B., et al. (2004). Composite cellulose fibers with magnetic properties. Kompozyty (Composites), 4, 36-40. 
[21] Kulpinski, P. (2007). Cellulose fibers modified by hydrophobic type polymer. Journal of Applied Polymer Science, 104, 398-409.

[22] Smiechowicz, E., Kulpinski, P., Bemska, J., Morgiel, J. (2014). Effect of silver nanoparticles shape, size and distribution on cellulose fibers' color. Coloration Technology, 130, 424-431.

[23] Skwierczynska, M., Runowski, M., Kulpinski, P., Lis, S. (2019). Modification of cellulose fibers with inorganic luminescent nanoparticles based on lanthanide(III) ions. Carbohydrate Polymers, 206, 742-748.

[24] Meister, F., Vorbach, D., Michels, C., Maron, R., Berghof, K., Taeger, E. (1998). Lyocell products with built-in functional properties. Chemical Fibers International, 48, 32-35.

[25] Stobinski, L., Lesiak, B., Malolepszy, A., Mazurkiewicz, B., Mierzwa, J., et al. (2014). Graphene oxide and reduced graphene oxide studied by the XRD, TEM and electron spectroscopy methods. Journal of Electron Spectroscopy and Related Phenomena, 195, 145-154.
[26] Stauffer, D., Aharony, A. (1994). Introduction to percolation theory. Taylor and Francis (London).

[27] Zallen, R. (2004). The physics of amorphous solids. WileyVCH (Weinheim).

[28] Stankovich, S., Dikin, D. A., Piner, R. D., Kohlhaas, K. A., SonBinh, Y. J., et al. (2007). Synthesis of graphene-based nanosheets via chemical reduction of exfoliated graphite oxide. Carbon, 45(7), 1558-1565.

[29] Kulpinski, P. (2005). Cellulose fibers modified by silicon dioxide nanoparticles. Journal of Applied Polymer Science, 98, 1793-1798.

[30] Chatterjee, K., Tabor, J., Ghosh, T. K. (2019). Electrically conductive coatings for fiber-based E-textiles. Fibers, 7(6), 51-97. 